\section{Apolipoprotein A-V}

K. J. Lackner ${ }^{1}$ und D. Peetz ${ }^{2}$

${ }^{1}$ Institut für Klinische Chemie und Laboratoriumsmedizin, Universitätsmedizin Mainz, Mainz, Deutschland

${ }^{2}$ HELIOS Klinikum Berlin-Buch Institut für Labormedizin, Berlin, Deutschland

\section{Synonym(e) ApoA-V}

\section{Englischer Begriff apolipoprotein A-V}

Definition Apolipoprotein der $>$ Chylomikronen, $>$ Very low density Lipoprotein und High Density Lipoproteine (s. - High Density Lipoprotein).

Beschreibung. ApoA-V ist ein $41 \mathrm{kDa}$ schweres $>$ Apolipoprotein, das vorwiegend in der Leber synthetisiert wird. Es ist in den Katabolismus triglyzeridreicher $>$ Lipoproteine über die Aktivierung der Lipoproteinlipase involviert. Verschiedene Mutationen des ApoA-V sind mit Hypertriglyzeridämien und dem kardiovaskulären Risiko assoziiert, wobei die Kausalität noch offen ist. Auch eine kausale Beziehung zur familiär kombinierten Hyperlipoproteinämie wird diskutiert. Die Serumkonzentrationen sind mit ca. 25-400 $\mu \mathrm{g} / \mathrm{L}$ niedriger als die anderer Apolipoproteine. Ihre Bestimmung erfolgt derzeit nur in klinischen Studien.

\section{Literatur}

Musunuru K, Kathiresan S, Surprises from genetic analyses of lipid risk factors for atherosclerosis (2016) Apolipoprotein A5 and triglyceridemia. Focus on the effects of the common variants. Circ Res 118:579-585 